\title{
Retrospective Evaluation of the Results of Elastic Intramedullary Nailing in Pediatric Femoral, Tibial, and Forearm Diaphyseal Fractures
}

\author{
Zekeriya Okan Karaduman $^{1}$ \\ ${ }^{1}$ Department of Orthopedics and Traumatolog, Faculty of Medicine, Duzce University, Düzce, Turkey \\ Received: 13 November 2019, Accepted: 05 December 2019, Published online: 31 December 2019 \\ (C) Ordu University Institute of Health Sciences, Turkey, 2019
}

\begin{abstract}
Objective: In this study, we aimed to evaluate the clinical and radiological outcomes of pediatric femur, tibia, and diaphyseal fractures treated with elastic intramedullary nails.

Method: Overall 50 patients (34 male and 16 females; mean age: 7.7 years; range 5-14 years) diagnosed with femur, tibia, and forearm diaphyseal fractures were treated with titanium elastic nails (TEN). Of these, 18 were femur, 16 were tibia, and 16 were forearm diaphyseal fractures. One femoral shaft fracture was fragmented, and one tibia fracture was an open fracture. Open reduction was performed in two patients, whereas closed reduction was performed in the other patients. Anteroposterior (AP) and Lateral radiographies were used in the follow-up of patients. The patients were evaluated according to the range of motion, ability to use extremities, hospitalization duration, union duration of the fractures, inequality that may occur in the extremities, and development of complications. Flynn scoring system was used for evaluating the cases.

Results: Union of the fractures was achieved in an average of 6.9 (range: 4-11) weeks.A patient had a tibia fracture in the front-back plane AP with 5 varus angulation, 10 posterior angulation with a forearm fracture in the lateral plane, and $2.5 \mathrm{~mm}$ shortness was observed in an another forearm fracture. All patients, except one, had normal range of motion and could comfortably use the extremities. No nonunion, epiphysial growth arrest, and infection was observed in any patient. According to the Flynn criteria; perfect results were achieved in 34 patients and successful results were achieved in 16. Average union duration was 9.6 (range: 5-14) weeks, hospitalization duration was 3.5 (range: 3-6) days, and follow-up duration was 12.4 (range: 619) months.

Conclusions: Titanium intramedullary elastic nail is a treatment method that can be successfully performed on pediatric femur, tibia, and forearm diaphyseal fractures.

Key words: Titanium intramedullary elastic nail, pediatric femur diaphysis fracture, pediatric tibia diaphysis fracture, pediatric forearm diaphysis fracture, surgery
\end{abstract}

Suggested Citation: Karaduman ZO. Retrospective Evaluation of the Results of Elastic Intramedullary Nailing in Pediatric Femoral, Tibial, and Forearm Diaphyseal Fractures. Middle Black Sea Journal of Health Science, 2019; 5(3):226-232.

Address for correspondence/reprints:

E-mail: karadumano@hotmail.com

Zekeriya Okan Karaduman

DOI: $\quad 10.19127 /$ mbsjohs.646579

Telephone number: +90 (505) 575-38-30 


\section{Introduction}

Pediatric fractures differ from adult fractures in terms of having more growth factor related environment, thicker periost, remodeling capacity, and less ligament injuries. These factors affect treatment approach. Many pediatric fractures are may be treated with conservative methods (Barry, 2004), However titanium elastic nail (TEN) are being used in the fixation of diaphysis long-bone fractures in adolescents and children (Lee, 2018).With great success. The biggest advantages of TEN are as follows: the minimally invasive nature of the technique, short operation time, and protection of the growth plate. Titanium and stainless steel are generally used in the production of elastic nails and both the metals have enough strength for adequate stabilization of the fractured bone (Mohamed, 2017).

In fractures that require operation, alternative treatment methods such as fixation with plate screw or external fixation may also be used successfully (Mohamed, 2017), however TEN allows slight movement in the fracture line and primary bone union without growth plate injury; it is a minimally invasive method that forms a small and esthetic scar tissue allowing early joint movement and normal physical activity, low infection rates, shortened psychological effects due to immobility, and shortened hospitalization duration, whichis the most important reasons that the method is widely accepted (Rajesh, 2016).

Intramedullary nailing in children with open epiphysis can be used in diaphyseal region fractures and some metaphyseal region fractures (Furlan, 2011) and additional immobilization with casting, usually not required postoperatively. In the present study, we aimed to evaluate the clinical and radiological outcomes of the titanium elastic nailing application performed on femur, tibia, and forearm diaphyseal fractures of children aged between 5-14 years.

\section{Methods}

The retrospective clinical study was approved by the Düzce University clinical research local ethics committee (decision numbered 236/2019). Overall 50 patients ( 34 male and 16 female; average age: 7.7,range 5-17 years)with 18 femur, 16 tibia, and 16 forearm diaphysis fracture diagnoses who were admitted to the emergency services at the Düzce University Faculty of Medicine Orthopedics and Traumatology clinic between May 1st, 2014 and October 31st, 2016, those aged > 5 and $<14$ years with femur, tibia, and forearm diaphysis fracture diagnosis who could not be stabilized with closed reduction and who were treated with TEN method were included. Sociodemographic and preoperative characteristics of the patients are shown in Table 1. All patients were treated with TEN.

Table 1. Sociodemographic and preoperative characteristics of patients

\begin{tabular}{lr}
\hline Preoperative Patient Data & \multicolumn{1}{c}{ Value } \\
\hline Number of patients & 50 \\
Male & $34(68 \%)$ \\
Female & $16(32 \%)$ \\
Average age (years) & $.7(5-14)$ \\
Etiology & \\
Simple falling & $14(28 \%)$ \\
Fall off of a bike & $15(30 \%)$ \\
Playing football & $13(26 \%)$ \\
Fall off the swing & $4(8 \%)$ \\
Traffic accident & $4(8 \%)$ \\
\hline Fracture side & \\
Right & $32(64 \%)$ \\
Left & $18(36 \%)$ \\
\hline
\end{tabular}

Surgical Tecnique: Closed reduction under Scopy Control was attempted in all patients. After reduction, physis lines were determined. In femur fractures, the lower end of the femur was reached with two incisions in distal lateral, and medial metaphyseal regions. Two TENs were sent retrograde from holes opened from the lateral and medial region of the lower end of the femur to the subtrochanteric region passing through the fracture line (Figure 1a, b). In cases where reduction could not be achieved, fracture site was detected, and reduction was provided with possible minimal disection. In cases where adequate stability was not achieved, one more elastic nail was sent from the lateral side.

In tibia fractures; medial and lateral mini incisions were performed on $2 \mathrm{~cm}$ distal to tibial proximal epiphysis two elastic nails were sent through these holes till to the tibiaL lower end so as to pass the fracture line (Figure 2a, b).

In forearm fractures, the entrance point was determined from the lateral end of the distal physis using scopy for radius fractures. One TEN was sent in retrograde fashion from the opened hole. In case of an ulna fracture, proximal physis line was determined using again scopy and the nail was sent in antegrade fashion from the hole opened at the olecranon distal to physeal line (Figure 3a, b). 


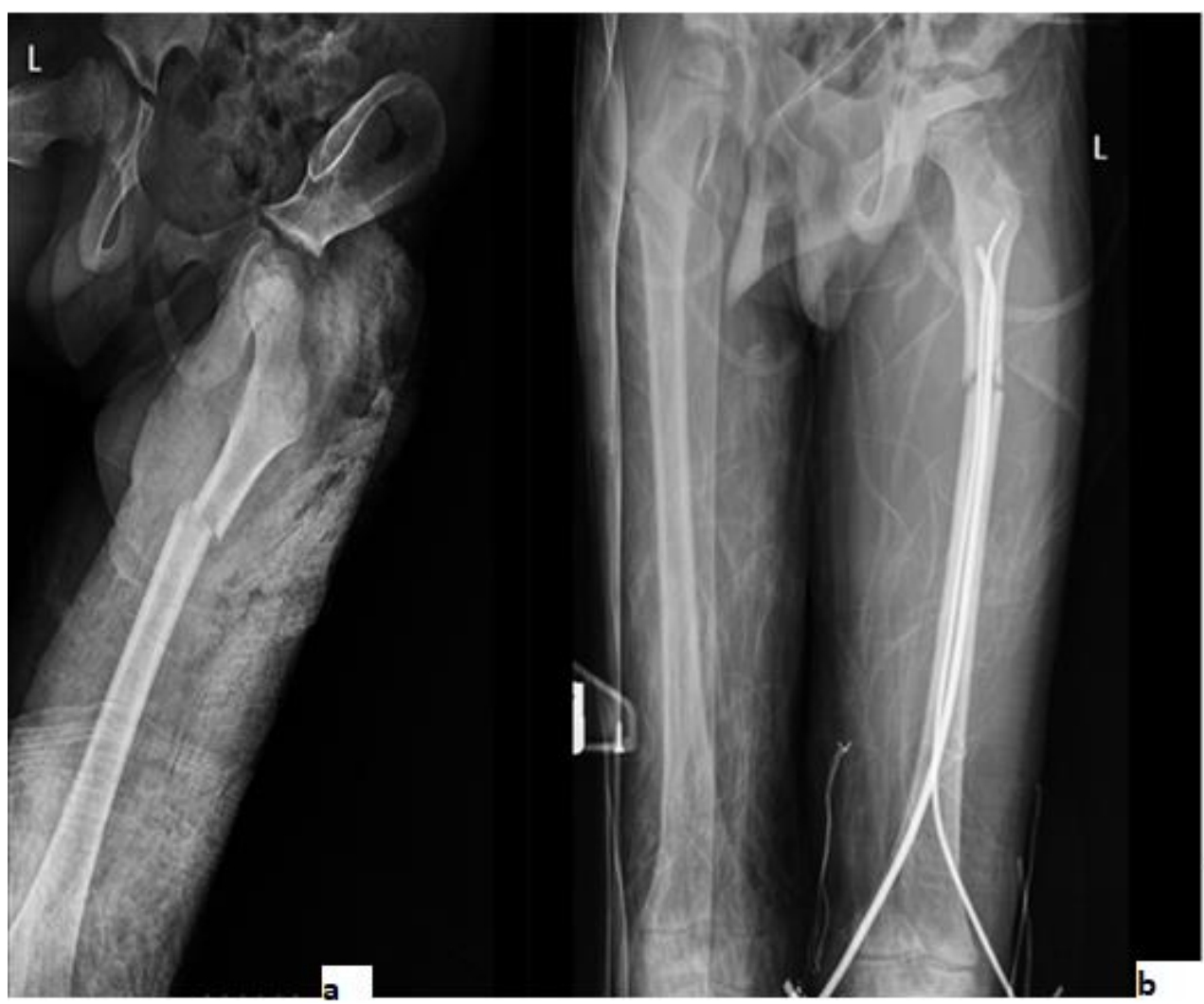

Figure 1a and b. Preoperative and Postoperative X-Ray Images of the Femur Fracture

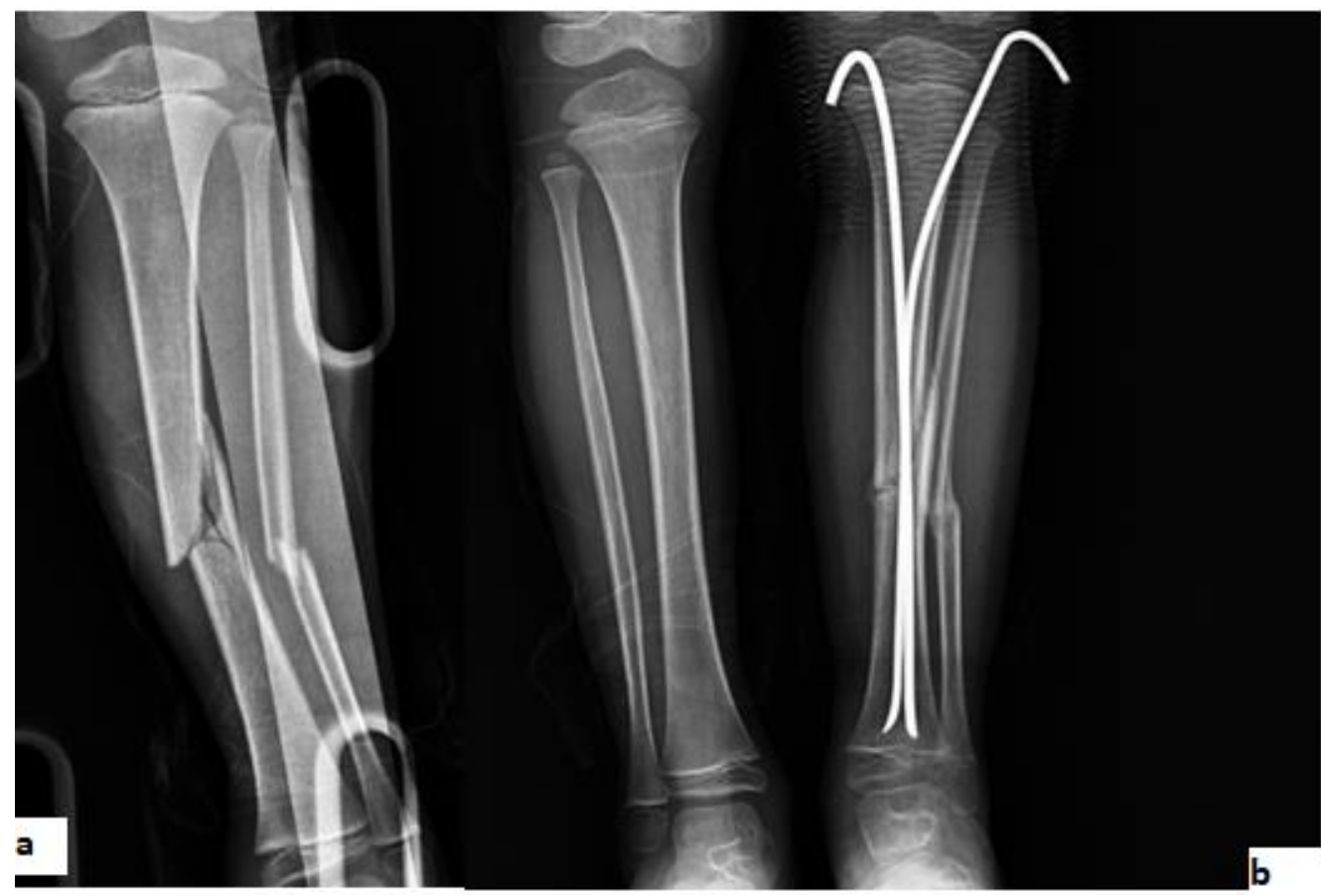

Figure 2a and b. Preoperative and Postoperative X-ray Image of the Tibia Fracture 


\section{Evaluation of Elastic Nailing Results in Pediatric Fractures}

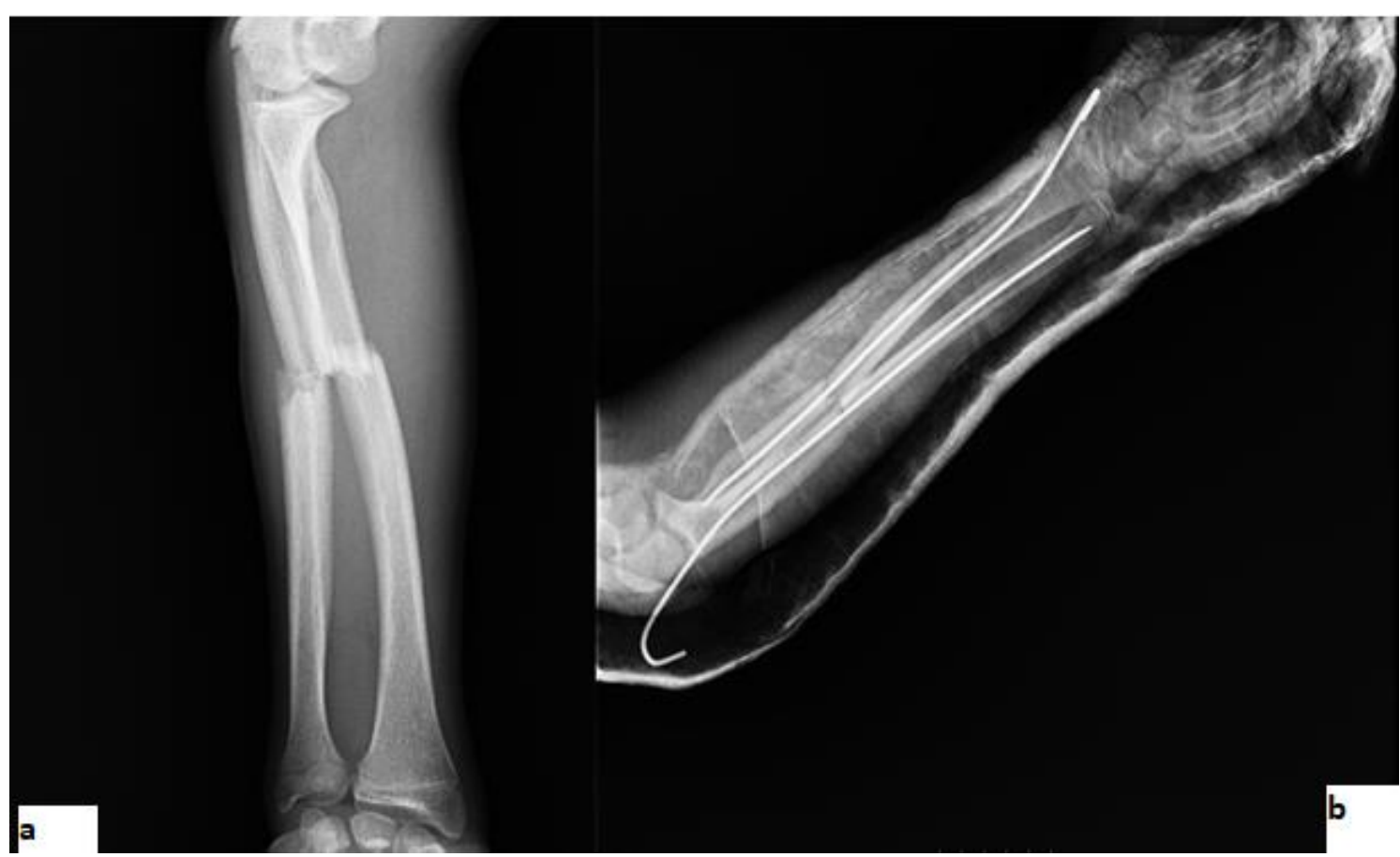

Figure 3a and b. Preoperative and postoperative X-ray images of the forearm fracture

Operated extremities of the patients were placed in braces for 2 weeks during the postoperative period. The patients were invited for routine followup at the postoperative $2 \mathrm{nd}, 4 \mathrm{th}, 8 \mathrm{th}$, and 16 th week and at 6th and 12th month. Ap-Lat radiographies were taken. Bony union decision was given when trabeculae or cortical bone crossing the fracture site was detected on radiographies and fracture site was stable and pain free during clinical examinations. The cases were evaluated in terms of hospital stay, fracture union duration, whether complications occurred, extremity length inequality, joint range of motion, and ability to use extremities. Clinical and radiological evaluation results were performed according to the titanium elastic nailing system of Flynn et al. (2001). This system was evaluated to have perfect, successful, and bad results according to length inequality $(<1,1-2$, and $>2 \mathrm{~cm})$, alignment $\left(<5^{\circ}, 5^{\circ}-10^{\circ}\right.$, and $\left.>10^{\circ}\right)$, pain (does not exist, does not exist, and exists), and complications (doesn't exist, minor-temporary, and major-permanent) (Table 2).

\section{Statistical Evaluation}

In our study, SPSS version 22.0 (SPSS, Chicago, Illinois, USA) was used for descriptive statistical methods (mean, standard deviation, frequency). These analyzes revealed that a sample of this size was sufficient to detect a clinically significant difference with $100 \%, 89 \%$, and $95 \%$ power. Significance level was set at $\mathrm{p}=0.05$.
Table 2. Flynn criteria (2001)

\begin{tabular}{llll}
\hline & $\begin{array}{l}\text { Perfect } \\
\text { result }\end{array}$ & $\begin{array}{l}\text { Good } \\
\text { result }\end{array}$ & Bad result \\
\hline Limb length & $<1.0$ & $1-2 \mathrm{~cm}$ & $>2.0 \mathrm{~cm}$ \\
discrepancy & $\mathrm{cm}$ & $5-10^{\circ}$ & $>10^{\circ}$ \\
Angular & $<5^{\circ}$ & No & Yes \\
deformity & No & Minor- & Major- \\
Pain & No & transitory & permanent \\
Complication & & & \\
& & & \\
\hline
\end{tabular}

\section{Results}

Of the fractures, 18 were femur, 16 were tibia, and 16 were forearm diaphyseal fractures. Union was achieved in all fractures in an average of 9.6 (range: 5-14) weeks. Average duration of hospital stay was 3.5 (range: 3-6) days. Average follow-up duration was 12.4 (range: 6-19) months.

One tibia fracture was an open fracture and one femur fracture was a comminuted fracture with butterfly fragment. Of the fractures, $14,15,13,4$, and 4 occurred due to simple falling, falling off of a bicycle, falling while playing football, falling off of a swing, and due to traffic accidents, respectively. There was head trauma in two cases. Open reduction was performed in two patients. A third elastic nail was sent from the lateral side in one femur fracture to provide adequate stability. Normal alignment was acquired in coronal and sagittal plane 
in 48 patients, whereas $5^{\circ}$ varus angulation was observed in a tibial bone fractured patient and $10^{\circ}$ posterior angulation was observed in one of the forearm fractured patient; $2.5 \mathrm{~mm}$ shortness was observed in an additional forearm fractured patient. Slight limitation of hip and knee range of motion was in one of the femur fractured patient which became normal at his control 6 months post operatively. Nails were extracted from all patients in an average of 8 (range: 5-12) months. No delay in union, closing of growth plate (Epiphysis), and infection were observed. According to Flynn criteria, perfect outcome was achieved in 42 patients $(84 \%)$ and successful outcome was achieved in eight $(16 \%)(\mathrm{p}=0.05)$.

\section{Discussion}

In the recent years, the treatment of pediatric long-bone fractures with intramedullary nailing has been increasing. This is due to the technological advancements of the applied nails along with the changes made especially in elastic nails with the dominant attitude of the orthopedists involved in pediatric fractures (Barry, 2004). Primary advantages of elastic nails are as follows: early return to school after surgery, short hospital stays, low complication rates, remodeling, and fast bone healing without damaging the blood circulation of the physical plaque (Heinrich, 1992; Öztürkmen, 2002). Although it seems difficult to provide rotational stability and alignment during the elastic nail application, a closed reduction application accompanied by scopy control and careful aligment control during surgery minimizes complications (Aktekin, 2007). In the present study, open reduction was performed only in one tibial open fracture and one femural fragmented fracture. The most important disadvantages of these nails are as follows: inadequacy in providing rotational stability, shortness risk in fragmented fractures, bending of the nails from the fracture line, and discomfort at the nail entrance point (Kawalkar, 2018). Gogi et al. (2007) followed up pediatric patients with femur shaft fractures who were operated with elastic nails. They reported an average of $2.7-\mathrm{mm}$ length increase in nine patients and an average of $11.7-\mathrm{mm}$ decrease in four. They did not encounter any leg-length inequality in 10 patients. They reported that leg-length inequality is commonly observed, and it does not lead to functional problems over time. In the present study, no shortness was observed in any patient with femur fractures. Limitation was observed in hip and knee movements in one patient. The functions of this patient recovered during the follow-ups. No rotational alignment disorder clinically was observed in any patient. The treatment performed with TENs may cause nail-path infection or irritation. In the study by Onta et al. (2015) and in another study by Sankar et al. (2007), it was reported that $22 \%$ and $26 \%$ patients, respectively, have pin-path irritation. Kapil et al. (Kapil, 2016) reported that $13.3 \%$ patients had pin-path irritation and $4.4 \%$ patients had superficial pin-path infection. In our study, the nails embedded under the skin and caused irritation in three $(6 \%)$ patients which were resolved by itself after the removal of TENs without causing any problems.

In pediatric forearm fractures, even the angulations $>20^{\circ}$ are corrected with remodeling children aged $<10$ years. However, in those aged $>8-10$ years, angulations $>10^{\circ}$ should not be accepted (Fuller, 1982; Larsen, 1988). Angulations $>10^{\circ}$ cause $3-4-\mathrm{mm}$ relative shortness in the angulated bone, functional loss in the distal radioulnar joint, disruption of the interosseous distance, and limitation in the rotation movement of the forearm (Slongo, 2005; Kubiak, 2005). In the present study, a $10^{\circ}$ posterior angulation was observed in one forearm fracture case and $2.5-\mathrm{mm}$ shortness was observed in another forearm fracture case. However, no limitations were observed in the forearm rotation and wrist movements of the patients.

Generally, pediatric isolated tibia fractures are treated with conservative methods. Surgical indication is indicated by selected open fractures that cannot be reduced with closed reduction; those with multiple trauma and fractures; those with spasticity such as cerebral palsy, extreme soft tissue trauma, and compartment syndrome, and in children aged >10 years (Aktekin, 2007). Intramedullary nailing is not widely used in pediatric tibia fractures due to the triangular structure of the tibia, proximal and distal tibiofibular joints being located within the eccentric muscle structure, and its curve at the proximal end (Slongo, 2005). In all pediatric fractures, tibia fractures are the only ones that developed nonunion and malunion with elastic nail treatment according to slongo et al. (Slongo, 2005). In a previous study, elastic nail treatment achieved good results in the less fragmented open tibia fractures, which do not have segmental bone loss (Kubiak, 2005). In another study,50 pediatric tibia fractures were treated with intramedullary nailsanddelayed union was observed in $11 \%$ and nonunion in $14 \%$ patients (Gordon, 2007). Moreover, they detected that delayed union and 
hypertrophic callous tissue formation increases with age. Leg-length inequality is the complication that may also occur after tibia diaphyseal fractures (Vallamshetla, 2006). They reported that 3.6\% patients had leg-length inequality of $<15 \mathrm{~mm}$. In our present study, shortness did not develop in any tibia shaft fractures. In pediatric tibia fractures, $10^{\circ}$ varus-valgus aligment in those aged $<8$ years and $5^{\circ}$ varus-valgus in those aged $>8$ years are acceptable (Bilge, 2008). In the present study, $5^{\circ}$ varus deformity developed in one patient but no problems were observed in their follow-ups.

A study evaluating 30 patients with tibia diaphyseal fractures reported that they achieved perfect, acceptable, and bad results at rates of 50\%, $36 \%$, and $14 \%$, respectively (Debnath, 2017). In a study comparing patients that had either TEN or plate-screw osteosynthesis, it was reported that $97 \%$ had similar recovery (Pennock, 2017). In a study evaluating 16 patients (O'Brien, 2004), it was reported that all fractures completely healed without any pain within a 5-year period and no limitation of movement or refractures was observed. Furthermore, another study evaluating 19 patients with stable fractures, perfect, acceptable, and bad results were reported in 63\%,32\%, and 5\% patients, respectively (Sankar, 2007). Uludağ et al. achieved perfect and successful results in $60 \%$ and $40 \%$ of their patients, respectively (Uludağ, 2019). Another study conducted on 48 children using Flynn criteria (Govindasamy, 2018), the clinical results were evaluated, and perfect results were achieved in 42 children $(83 \%)$ and adequate results were achieved in eight children (17\%). In the present study, according to the Flynn criteria, perfect results were achieved in 42 patients (84\%) and successful results in $8(16 \%)$.

Using the elastic nail method, hospital stays, and related treatment costs significantly decreased according to Bilge et al. (Bilge, 2008). Average duration of hospital stays for our patients were 3.5 (range: 3-6) days and average follow-up duration was 12.4 (range: 6-9) months. In all fractures, union was achieved in an average of 9.6 (range: 5-14) weeks. In cases with plate application after pediatric long-bone fracture, plate needs to be removed after the fracture union. There is also a higher risk of refracture development after the removal of the plate This operation also causes relatively same amount of bleeding and trauma as the first operation. Moreover, due to the re-fracture risk after plate removal, patients are usually asked to use crutches for at least 3 weeks after removal. Thus, the activities of the patients during this period are limited due to the possibility of fracture. An important advantage of the TEN application is that the re-fracture risk is much lower than the plate application.

Intramedullary elastic nail application is an effective method used in pediatric long-bone fracture treatment. We believe that good results can be achieved with correct patient selection, appropriate surgical technique, and a good patient follow-up.

\section{Conclusion}

TEN can be successfully applied as a safe, effective, and low complication method in the treatment of pediatric femur, tibia, and forearm diaphyseal fractures aged between 5-12 years.

Ethics Committee Approval: Ethics committee approval was received for this study from Düzce University clinical research local ethics committee (decision numbered 236/2019)

Peer-review: Externally peer-reviewed.

Author Contributions: Concept- ZOK.; Design ZOK.; Supervision- ZOK.; Materials ZOK.; Data Collection and/or Processing ZOK.; Analysis and/or Interpretation- ZOK.; Literature ReviewZOK.; Writing- ZOK.; Critical Review-ZOK.

Conflict of Interest: No conflict of interest was declared by the author.

Financial Disclosure: The author declared that this study hasn't received no financial support.

\section{References}

Aktekin CN, Bicimoglu A. Intramedullary nailing in pediatric fractures. Totbid Dergisi 2007; 6(34): 102-8.

Aktekin CN, Ozturk AM, Altay M, Toprak A, Ozkurt B, Tabak AY. Flexible intramedullary nailing of children. Ulus Travma Acil Cerrahi Dergisi 2007; 13: 115-21.

Barry M, Paterson JM. A flexible intramedullary nail forfractures in children. J Bone JointSurgBr 2004; 86(7): 947-53.

Bilge O, Aksoy CM. Fractures of tibia in children Totbid dergisi 2008; 7(3-4): 121-6.

Debnath S, Debbarma S, Sarkar A. Titanium elastic nailing osteosynthesis for diaphyseal tibial fracture in pediatric age group-our experience. Indian J. Appl. Res 2017; 7: 52-53. 


\section{Evaluation of Elastic Nailing Results in Pediatric Fractures}

Flynn JM, Hresko T, Reynolds RA, et al. Titanium elastic nails for pediatric femur fractures: A multicenter study of early results with analysis of complications. J Pediatr Orthop 2001; 21: 48.

Fuller DJ, McCullough CJ. Malunited fractures of the forearm in children. J Bone JointSurg (Br). 1982; 64: 364-7.

Gogi N, Khan SA, Varshney MK. Limb lenght discrepancy following titanium elastic nailing pediatric femoral shaft fractures. ActaOrthopBelg 2006; 72: 154-8.

Gordon JE, Gregush RV, Schoenecker PL, Dobbs MB, Luhmann SJ. Complications after titanium elastic nailing of pediatric tibial fractures $\mathrm{J}$ Pediatr Orthop 2007; 27(4): 442-6.

Govindasamy R, Gnanasundaram R, Kasirajan S, Ibrahim S, Melepuram JJ. ElasticStableIntramedullaryNailing of FemoralShaftFracture-Experience in 48 Children. Arch Bone JtSurg. 2018;6(1):39-46.

Heinrich SD, Drvaric D, Darr K, MacEwen GD. Stabilization of pediatric diaphyseal femur fractures with flexible intramedullary nails (a techniquepaper). J OrthopTrauma 1992; 6(4): 452-9.

Kc KM, Acharya P, Sigdel A. Titanium Elastic Nailing System (TENS) for Tibia Fractures in Children: Functional Outcomes and Complications. JNMA J Nepal Med Assoc. 2016; 55(204): 55-60.

Kubiak EN, Egol KA, Scher D, Wasserman B, Feldman D, Koval KJ. Operative treatment of tibialfractures in children: Are elastic stable intramedullary nails an improvement over external fixation? J Bone JointSurg Am 2005; 87(8): 1761-8.

Larsen E, Vittas D, Trop-Pedersen S. Remodeling of angulated distal forearm fractures in children. ClinOrthop 1988; 237: 190-5.

O'Brien T, Weisman D.S, Ronchetti P, Piller C.P, Maloney M. Flexible Titanium Nailing for the Treatment of the Unstable Pediatric Tibial Fracture. J. Pediatr. Orthop 2004; 24: 601-609.

Onta P.R, Thapa P, Sapkota K, Ranjeet N, Kishore A. Gupta, M. Outcome of diaphyseal fracture of tibia treated with flexible intramedullary nailing in pediatrics age group; A prospective study. Am. J. Public Health 2015; 3: 65-68.

Ozturkmen Y, Dogrul C, Balioglu MB, Karli M. Intramedullery stabilization of pediatric diaphyseal femurfractures with elastic Ender nails. Acta Orthop Traumatol Turc 2002; 36: 220-7.
Pennock A.T, Bastrom T.P, Upasani V.V. Elastic intramedullary nailing versus open reduction internal fixation of pediatric tibial shaft fractures. J. Pediatr. Orthop 2017; 37: 1.

Sankar W.N, Jones K.J, David Horn B, Wells L. Titanium elastic nails for pediatric tibial shaft fractures. J. Child. Orthop 2007; 1: 281-286.

Slongo TF. Complicationsandfailures of the ESIN technique. Injury 2005; 36: 78-85.

Uludag A, Tosun HB. Treatment of Unstable Pediatric Tibial Shaft Fractures with Titanium Elastic Nails. Medicina (Kaunas) 2019; 10: 55(6).

Vallamshetla VR, De Silva U, Bache CE, Gibbons PJ. Flexible intramedullary nails for unstable fractures of the tibia in children. An eight-year experience. J Bone Joint Surg Br. 2006; 88(4): 536-40. 